\title{
LOGICAL AND ALGORITHMIC PROPERTIES OF INDEPENDENCE AND THEIR APPLICATION TO BAYESIAN NETWORKS *
}

\author{
Dan GEIGER and Judea PEARL \\ Cognitive Systems Laboratory, Computer Science Department, University of California, Los Angeles, \\ CA 90024, USA
}

\begin{abstract}
This paper establishes a partial axiomatic characterization of the predicate $I(X, Z, Y)$, to read " $X$ is conditionally independent of $Y$, given $Z$ ". The main aim of such a characterization is to facilitate a solution of the implication problem namely, deciding whether an arbitrary independence statement $I(X, Z, Y)$ logically follows from a given set $\Sigma$ of such statements. In this paper, we provide a complete axiomatization and efficient algorithms for deciding implications in the case where $\Sigma$ is limited to one of four types of independencies: marginal independencies, fixed context independencies, a recursive set of independencies or a functional set of independencies. The recursive and functional sets of independencies are the basic building blocks used in the construction of Bayesian networks. For these models, we show that the implication algorithm can be used to efficiently identify which propositions are relevant to a task at hand at any given state of knowledge. We also show that conditional independence is an Armstrong relation [10], i.e., checking consistency of a mixed set of independencies and dependencies can be reduced to a sequence of implication problems. This property also implies a strong correspondence between conditional independence and graphical representations: for every undirected graph $G$ there exists a probability distribution $P$ that exhibits all the dependencies and independencies embodied in $G$.
\end{abstract}

Keywords: Bayesian networks, conditional independence, graphoids, graphical representation, probabilistic reasoning.

\section{Introduction}

The role of conditional independence stems from several practical considerations. First, knowledge about independencies saves space when storing an explicit distribution function (e.g., by a table), and saves time when computing and updating the probability of an event. For example, in the extreme case of representing the distribution of $n$ independent binary variables, ignoring the independencies would require an explicit table of $2^{n}$ entries, and to calculate $P(x$

* This work was partially supported by the National Science Foundation Grant \#IRI-8610155. "Graphoids: A Computer Representation for Dependencies and Relevance in Automated Reasoning". 
is true) would require a summation over the other $n-1$ variables in the table. Recognizing the independencies among the variables enables us to replace the table by $n$ parameters and to reduce computations to a single operation. Second, if we choose to represent and process random variables by networks [28], then the topology of such networks must reflect the conditional independencies that govern the variables in the domain and, therefore, the set of transformations [33] we are permitted to apply to the networks must, likewise, be determined by the rules that govern conditional independence. Third, in eliciting probabilistic models from human experts, dependencies among variables can often be asserted qualitatively while numerical assessments are subjected to a great deal of uncertainties. Obtaining a direct representation scheme for judgements about dependencies would guard the model builder from assigning numerical values that lead to conceptually unrealistic dependencies. Examples of such representation schemes are graphical dependency models such as Undirected Graphs (UG, section 3) $[18,21,26]$, Directed Acyclic Graphs (DAG, section 4) $[16,19,22,28,33]$, or Recursive models $[20,41]$.

In this paper we concentrate on probabilistic conditional independence (although some results extend to other definitions of independence) because of its role in probabilistic reasoning systems (such as $[1,4,7]$ ). Information about dependencies can be specified by a list of independence statements (or simply statements $)$ of the form $I(X, Z, Y)_{P}$ where, $X, Y$ and $Z$ are three finite disjoint * sets of variables and $I(X, Z, Y)_{P}$ stands for " $X$ is conditionally independent of $Y$, given $Z$ ", or equivalently,

$I(X, Z, Y)_{P} \Leftrightarrow P(x, y \mid z)=P(x \mid z) \cdot P(y \mid z)$,

for any instantiation $x, y$ and $z$ of the variables $X, Y$ and $Z$, for which $P(z)>0$. (By convention, we assume that $I(X, Z, \varnothing)$ always holds.) When an independence statement does not hold we say it is a dependency.

Our objective is to answer the following three questions:

1. The implication problem: Does an independence statement $\sigma$ logically follow from a set of such statements $\Sigma$, namely, does $\sigma$ hold in very distribution that obeys all statements in $\Sigma$ ?

2. Given a set of independence statements, is the set redundant, i.e., are some of the statements implied by the others?

3. Given a set of independence statements $\Sigma^{+}$and a set of dependence statements $\Sigma^{-}$, is the combined set $\Sigma^{+} \cup \Sigma^{-}$consistent, i.e., could it be realized simultaneously by some probability distribution?

The last two problems are reducible to a sequence of implication problems. To answer the second question we simply select each statement $\sigma$ in $\Sigma$ and check whether $\sigma$ logically follows from $\Sigma-\{\sigma\}$. The answer to the third problem is affirmative iff $\Sigma^{+}$does not imply the negation of any dependency in $\Sigma^{-}$. The

\footnotetext{
* An assumption used for clarity of presentation.
} 
correctness of this reduction stems from the fact that independence is an Armstrong relation as shown in section 2.

The most common strategy for solving the implication problem involves a two step process [2,3,8]; first finding a complete set of inference rules called axioms, and second, finding an efficient algorithm that repeatedly applies these axioms to determine whether $\sigma$ is derivable from $\Sigma$. Completeness guarantees that, by repeated application of the axioms, each statement $\sigma$ that logically follows from $\Sigma$ will eventually be derived. Examples of axioms for conditional independence are [28]:

$\begin{array}{ll}\text { Symmetry } & I(X, Z, Y) \Rightarrow I(Y, Z, X), \\ \text { Decomposition } & I(X, Z, Y \cup W) \Rightarrow I(X, Z, Y), \\ \text { Weak union } & I(X, Z, W \cup Y) \Rightarrow I(X, Z \cup W, Y), \\ \text { Contraction } & I(X, Z, Y) \& I(X, Z \cup Y, W) \Rightarrow I(X, Z, Y \cup W) .\end{array}$

These axioms are used in [30,31] to generalize the concept of independence; any ternary predicate obeying axioms (1.a) through (1.d) is called a semi-graphoid [31]. Other examples of semi-graphoids include Partial Correlation [5,30], Ordinal Conditional Functions [17,37], Embedded Multivalued Dependencies [9], Qualitative Independence [34], Vertex Separation [30], $d$-separation [28] and $D$-separation [15] (hence the name semi-graphoid). Similar axioms for probabilistic independence were used by Dawid [6], Spohn [36], and Smith [35].

In this paper we carry out these two steps for four classes of independence statements: marginal independence (section 2), fixed context independence (section 3) and both a recursive and a functional set of independence (section 4) (for which axioms (1) are shown to be complete). The general implication problem for unrestricted sets of conditional independence statements $\Sigma$, remains unsolved. The results reported in [23,38] suggest that a finite complete set of axioms for conditional independence does not exists. This, however, does not exclude a possible solution to the implication problem because a non-axiomatizable set of statements can still admit an efficient implication algorithm (e.g., [32]), but the existence of such a solution is less likely [39].

\section{Some completeness results}

The following notations are employed; $\sigma$, possibly scripted, denotes an independence statement, $\boldsymbol{\Sigma}$ denotes a set of independence statements and $\mathbf{P}$ denotes a class of probability distributions, for example strictly positive distributions $\left(P D^{+}\right)$, normal distributions $(P N)$, distributions over binary variables $(P B)$ and the class of all probability distributions $(P D)$. 


\section{DEFINITION}

An axiom

$\sigma_{1} \& \sigma_{2} \& \ldots \& \sigma_{n} \Rightarrow \sigma$

is sound for $\mathbf{P}$ if every distribution $P \in \mathbf{P}$ that obeys the antecedents of the axiom also obeys $\sigma$. Axioms (1.a) through (1.d) are examples of sound axioms for $P D$.

\section{DEFINITION}

$\sigma$ is logically implied (logically follows) by $\Sigma$, denoted $\Sigma \vDash_{\mathbf{P}} \sigma$, if every distribution in $\mathbf{P}$ that obeys $\Sigma$ also obeys $\sigma . \Sigma \vDash_{\mathbf{A}} \boldsymbol{\sigma}$ iff $\sigma \in \mathbf{c l}_{\mathbf{A}}(\Sigma)$, i.e., there exists a derivation chain $\sigma_{1}, \ldots, \sigma_{n}=\sigma$ such that for each $\sigma_{j}$, either $\sigma_{j} \in \Sigma$, or $\sigma_{j}$ is derived by an axiom in $\mathbf{A}$ from the previous statements.

\section{DEFINITION}

A set of axioms $\mathbf{A}$ is sound for $\mathbf{P}$ iff for every statement $\boldsymbol{\sigma}$ and every set of statements $\Sigma$

$\Sigma \vDash_{\mathbf{A}} \sigma$ only if $\Sigma \vDash_{\mathbf{P}} \sigma$.

The set $\mathbf{A}$ is complete for $\mathbf{P}$ iff

$\Sigma \vDash_{\mathbf{A}} \sigma$ if $\Sigma \vDash_{\mathbf{P}} \sigma$.

\section{PROPOSITION 1}

A set of axioms is sound for $\mathbf{P}$ iff each axiom in the set is sound for $\mathbf{P}$.

The proof is achieved by induction on the length of a derivation.

PROPOSITION 2 (After Fagin [8])

A set of axioms $\mathbf{A}$ is complete iff for every set of statements $\Sigma$ and every statement $\sigma \notin \mathbf{c l}_{\mathbf{A}}(\Sigma)$ there exists a distribution $P_{\sigma}$ in $\mathbf{P}$ that satisfies $\Sigma$ and does not satisfy $\sigma$.

Proof

This is the counter-positive form of the completeness definition, if $\sigma \notin \mathbf{c l}_{\mathbf{A}}(\Sigma)$ (i.e., $\left.\Sigma \nvdash_{\mathbf{A}} \sigma\right)$ then $\Sigma \nvdash_{\mathbf{P}} \sigma$.

Next we present a complete set of axioms for the class of marginal independence statements.

\section{DEFINITION}

A marginal statement (or marginal independency) is an independence statement $I(X, Z, Y)$ where $Z$ is $\varnothing$, i.e., $I(X, \varnothing, Y)_{P}$ iff $P(X, Y)=P(X) \cdot P(Y)$,

for each instantiation of $X$ and $Y$. The inequality $P(X, Y) \neq P(X) \cdot P(Y)$ is called a marginal dependency. 
THEOREM 3 (Completeness for marginal independence) [12]

Let $\Sigma$ be a set of marginal statements, and let $\operatorname{cl}(\Sigma)$ be the closure of $\Sigma$ under the following axioms:

Trivial independence $I(X, \varnothing, \varnothing)$.

Symmetry $I(X, \varnothing, Y)_{P} \rightarrow I(Y, \varnothing, X)_{P}$.

Decomposition $I(X, \varnothing, Y \cup W)_{P} \rightarrow I(X, \varnothing, Y)_{P}$.

Mixing $I(X, \varnothing, Y)_{P} \& I(X \cup Y, \varnothing, W)_{P} \rightarrow I(X, \varnothing, Y \cup W)_{P}$.

Then for every marginal statement $\sigma=I(X, \varnothing, Y)_{P} \notin \mathbf{c l}(\Sigma)$ there exists a probability distribution $P_{\sigma}$ that obeys all statements in $\operatorname{cl}(\Sigma)$ but does not obey $\sigma$.

\section{Proof (sketch)}

Let $\sigma=I(X, \varnothing, Y)$ be a statement not in $\mathbf{c l}(\Sigma)$ where $X=\left\{x_{1}, \ldots, x_{l}\right\}$ and $Y=\left\{y_{1}, \ldots, y_{m}\right\}$. Assume $\sigma$ is minimal, i.e. that for all nonempty sets $X^{\prime} Y^{\prime}$ obeying $X^{\prime} \subseteq X, Y^{\prime} \subseteq Y$ and $X^{\prime} Y^{\prime} \neq X Y$, we have $I\left(X^{\prime}, \varnothing, Y^{\prime}\right) \in \operatorname{cl}(\Sigma)$. We construct $P_{\sigma}$ as follows: Let the variables in $\Sigma \cup\{I(X, \varnothing, Y)\}$, except $x_{1}$, be independent binary variables with probability $\frac{1}{2}$ to each of their two values (e.g., fair coins) and let

$x_{1}=\sum_{i=2}^{l} x_{i}+\sum_{i=1}^{m} y_{J}(\bmod 2)$.

It is shown in [12] that $P_{\sigma}$ satisfies the requirement of the theorem. If $\sigma$ is not minimal, then we delete variables from $X$ and $Y$ until we obtain a minimal statement $\sigma^{\prime}=I(\hat{X}, \varnothing, \hat{Y})$ where $\hat{X} \subseteq X$ and $\hat{Y} \subseteq Y$. Then we construct $P_{\sigma^{\prime}}$ as above. $P_{\sigma^{\prime}}$ does not satisfy $\sigma^{\prime}$. By the decomposition axiom, $P_{\sigma^{\prime}}$ does not satisfy $\sigma$ either (while satisfying $\mathbf{c l}(\Sigma)$ ). Thus, $P_{\sigma^{\prime}}$ satisfies the requirements of the theorem.

\section{PROPOSITION 4}

Axioms (2) are sound for $P D$ (i.e., hold for all distributions).

The proof of Proposition 4 rests on the basic definition of independence. For example, to prove (2.d) we observe that $P(X, Y)=P(X) \cdot P(Y)$ and $P(X, Y, Z)$ $=P(X, Y) \cdot P(Z)$ imply that $P(X, Y, Z)=P(X, Y, Z)$. Moreover, summing over $X$ yields $P(Y, Z)=P(Y) \cdot P(Z)$, hence $P(X, Y, Z)=P(X) \cdot P(Y, Z)$, which establishes the right-hand side of (2.d).

Theorem 3 and Proposition 4 guarantee that by repeatedly applying axioms (2.a) through (2.d) on a set of marginal statements $\Sigma$, any marginal statement $\sigma$ that logically follows from $\Sigma$ will eventually be derived and, conversely, any marginal statement that is derivable, logically follows from $\Sigma$. Paz [12,24] provides an efficient algorithm to check whether a marginal statement $\sigma$ is 
derivable from $\Sigma$. The complexity of his algorithm is $O\left(|\Sigma| \cdot n^{2}\right)$ where $n$ is the number of distinct variables in $\Sigma \cup\{\sigma\}$.

A complete set of axioms does not provide sufficient means for deriving all the information that is implied by a given set of statements. For example, assume that the set $\Sigma=\{I(X, \alpha, Y), I(X, \varnothing, Y)\}$ is given, where $\alpha$ is a single variable and all variables are bivalued i.e., drawn from $P B$. It can be shown that the disjunction $I(X, \varnothing, \alpha)$ or $I(Y, \varnothing, \alpha)$ logically follows from $\Sigma$ and, yet, it cannot be derived by a complete set of axioms. Such a set only guarantees to reveal (correctly) that neither of the disjuncts is logically implied by $\Sigma$ but would not show that one of the two statements must hold. To obtain all disjunctions, a strongly complete set of axioms is needed.

DEFINITION (After $[3,8]$ )

A set of axioms $\mathbf{A}$ is strongly complete in a class of distribution $\mathbf{P}$, if for every set of statements $\Sigma$ and for every set of single statements $\left\{\sigma_{\imath} \mid i=1, \ldots, n\right\}$ the following relation holds:

$\Sigma \vDash_{\mathrm{P}} \sigma_{1}$ or ... or $\sigma_{n}$ iff $\Sigma \vdash_{\mathrm{A}} \sigma_{1}$ or ... or $\sigma_{n}$

Similar to Proposition 2, the following holds:

PROPOSITION 5 (After [3,8])

A set of axioms A is strongly complete iff for every set of statements $\Sigma$ closed under axioms $\mathbf{A}$, there exists a distribution $P$ in $\mathbf{P}$ that satisfies all statements in $\Sigma$ and none other.

Clearly, a complete set of axioms is strongly complete but the converse is not always true [8]. The notion of Armstrong relation [10] provides a condition under which strong completeness is equivalent to completeness.

\section{DEFINITION}

Conditional independence is an Armstrong relation in a class of distributions $\mathbf{P}$ if there exists an operation $\otimes$ that maps finite sequences of distributions of $\mathbf{P}$ into a distribution of $\mathbf{P}$, such that if $\sigma$ is a conditional independence statement and if $P_{l}, i=1 \ldots n$ are distributions in $\mathbf{P}$, then $\sigma$ holds for $\otimes\left\{P_{\imath} \mid i=1 \ldots n\right\}$ iff $\sigma$ holds for each $P_{l}$.

We concentrate on two families of distributions P: All distributions, denoted $P D$ and strictly positive distributions, denoted $P D^{+}$.

\section{THEOREM 6 [14]}

Conditional independence is an Armstrong relation in $P D$ and in $P D^{+}$.

The operation $\otimes$ is realized by the direct product. Let $P_{1}$ and $P_{2}$ be two distributions sharing the variables $x_{1}, \ldots, x_{n}$. Let $A_{1}, \ldots, A_{n}$ be the domains of 
$x_{1}, \ldots, x_{n}$ in $P_{1}$ and let $\alpha_{1}, \ldots, \alpha_{n}$ be an instantiation of these variables. Similarly, let $B_{1}, \ldots, B_{n}$ be the domains of $x_{1}, \ldots, x_{n}$ in $P_{2}$ and $\beta_{1}, \ldots, \beta_{n}$ an instantiation of these variables. Let the domain of $P=P_{1} \otimes P_{2}$ be the product domain $A_{1} B_{1}, \ldots, A_{n} B_{n}$ and denote an instantiation of the variables of $P$ by $\alpha_{1} \beta_{1}, \ldots, \alpha_{n} \beta_{n}$. Define $P_{1} \otimes^{\prime} P_{2}$ by the following equation:

$P\left(\alpha_{1} \beta_{1}, \alpha_{2} \beta_{2}, \ldots, \alpha_{n} \beta_{n}\right)=P_{1}\left(\alpha_{1}, \alpha_{2}, \ldots, \alpha_{n}\right) \cdot P_{2}\left(\beta_{1}, \beta_{2}, \ldots, \beta_{n}\right)$.

The construction of $\otimes$ for more than two distributions and the proof that $\otimes$ satisfies the definitions of Armstrong relations, uses only the definition of independence and can be found in [14].

An immediate application of strong completeness is the reduction of the consistency problem to a set of implication problems.

\section{DEFINITION}

A set of marginal dependencies $\Sigma^{-}$and a set of marginal independencies $\Sigma^{+}$ are consistent iff there exists a distribution that satisfies $\Sigma^{+} \cup \Sigma^{-}$. The task of deciding whether a set is consistent is called the consistency problem.

The following algorithm answers whether $\Sigma^{+} \cup \Sigma^{-}$is consistent: For each member of $\Sigma^{-}$determine, using the implication algorithm, whether its negation logically follows from $\Sigma^{+}$. If the answer is negative for all members of $\Sigma^{-}$, then the two sets are consistent, otherwise they are inconsistent.

The correctness of the algorithm stems from the fact that if the negation of each member $\sigma$ of $\Sigma^{-}$does not follow from $\Sigma^{+}$i.e., each member of $\Sigma^{-}$is individually consistent with $\Sigma^{+}$, then there is a distribution $P_{\sigma}$ that realizes $\Sigma^{+}$ and $\neg \sigma$. The distribution $P=\otimes\left\{P_{\sigma} \mid \neg \sigma \in \Sigma^{-}\right\}$then realizes both $\Sigma^{+}$and $\Sigma^{-}$, therefore the algorithm correctly identifies that the sets are consistent. In the other direction, namely when the algorithm detects an inconsistent member of $\Sigma^{-}$, the decision is obviously correct.

\section{A graph-based closure algorithm}

In this section we concentrate on another class of statements called $U$-statements.

\section{DEFINITION}

Let $U$ be a set of variables (the universe). A statement $I(X, Z, Y)$ is called a $U$-statement if $X \cup Y \cup Z=U$. A set of $U$-statements is called fixed-context.

The interest in this set of statements stems from two reasons. First, for the class of strictly positive distributions, $U$-statements obey the same axioms as vertex separation in undirected graphs. Second, there exists a compact representa- 


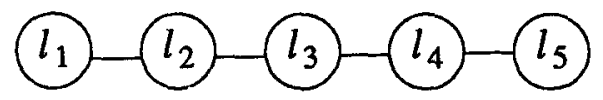

Fig. 1. Example of a Markov chain.

tion of all independence statements that logically follows (in $P D^{+}$) from a given set of $U$-statements, requiring only $O\left(|U|^{2}\right)$ bits of storage. These two properties rely on an additional axiom of independence that holds in every strictly positive distribution:

Intersection $I(X, Z \cup Y, W) \& I(X, Z \cup W, Y) \Rightarrow I(X, Z, Y \cup W)$.

(That intersection does not hold in all distributions can be seen by examining the case, $X=Y=W$ and $Z=\varnothing$.)

To exemplify the representation of probabilistic knowledge by undirected graphs, consider a language governed by a Markov process, namely, the probability of the $i$ th letter is determined solely by the $(i-1)$ th letter via $P\left(l_{i} \mid l_{t-1}\right)>0$. The dependencies embedded in the distribution function can be represented by the Markov chain of fig. 1.

This graph asserts, for example, that the variables $l_{1}$ and $l_{3}$ are conditionally independent given $l_{2}$, since the node $l_{2}$ blocks all paths from $l_{1}$ to $l_{3}$. More generally, for every three disjoint sets $X, Y$ and $Z$ of nodes in graph $G$, let us define the predicate $I(X, Z, Y)_{G}$ by

$I(X, Z, Y)_{G} \Leftrightarrow Z$ separates $X$ from $Y$ in $G$.

We then say that $G$ perfectly represents the dependencies of $P$ if there exists a 1-1 correspondence between the variables in $P$ and the vertices of $G$ such that, $I(X, Z, Y)_{G} \Leftrightarrow I(X, Z, Y)_{P}$.

Such a graph is called a perfect-map of $P$ [28].

Suppose that by sampling 5-letter words from some unknown language (see previous example), the following two independencies ( $U$-statements) were identified:

$\Sigma=\left\{I\left(\left\{l_{1}, l_{2}\right\}, l_{3},\left\{l_{4}, l_{5}\right\}\right), I\left(l_{3},\left\{l_{2} l_{4}\right\},\left\{l_{1} l_{5}\right\}\right)\right\}$.

The question arises: Are these statements sufficient to guarantee the Markov nature of the language and, moreover, is the chain structure a complete representation of all independencies that logically follow from $\Sigma$ ? The main result of this section is a polynomial-time algorithm that generates all independence statements that logically follow (in $P D^{+}$) from a set of $U$-statements $\Sigma$, i.e., it generates all statements that hold in every strictly-positive distribution which obeys $\Sigma$. In particular it reveals that $\Sigma$ implies the Markov nature of the language via $P\left(l_{i} \mid l_{i-1}, l_{i-2}, \ldots, l_{1}\right)=P\left(l_{i} \mid l_{i-1}\right) \quad i=2, \ldots, 5$,

or, in our notation, that $I\left(l_{1}, l_{i-1},\left\{l_{i-2} \ldots l_{1}\right\}\right)$ must hold for $i=2, \ldots, 5$ and, moreover, that no other statement is logically implied by $\Sigma$. This closure al- 
gorithm generates from $\Sigma$ the graph of fig. 1. It starts with a complete graph (over all the variables) and simply deletes every edge $(\alpha, \beta)$ for which a statement of the form $I(X \cup\{\alpha\}, Z, Y \cup\{\beta\})$ is found in $\Sigma$, for some $X, Y$ and $Z$. We show that the resulting graph represents all the independencies that are shared by all strictly-positive distributicis that obey $\Sigma$, and must therefore be obeyed by the language. Thus the two statements of $\Sigma$ constitute a sufficient code for the chain structure of the distribution $P$, and the algorithm uncovers this structure without resorting to numerical calculations. The next two theorems justify this algorithm.

THEOREM 7 [14]

For every undirected graph $G$, there exists a non-extreme distribution $P$, and a 1-1 correspondence between the variables in $P$ and the nodes of $G$ such that for every three disjoint sets of nodes $X, Y$ and $Z$ the following holds:

$I(X, Z, Y)_{G}$ iff $I(X, Z, Y)_{P}$.

\section{THEOREM 8 (strong completeness) $[14,25]$}

Let $\Sigma$ be a set of $U$-statements and let $\mathbf{c l}(\Sigma)$ be the closure of $\Sigma$ under symmetry (1.a), decomposition (1.b), weak union (1.c) and intersection (3). Then, (1) there exists an undirected graph $G$ for which $I(X, Z, Y)_{G}$ satisfies exactly the statements in $\operatorname{cl}(\Sigma)$, and (2) there exists a strictly positive distribution $P$ that satisfies exactly the statements in $\operatorname{cl}(\Sigma)$ (i.e., $G$ is a perfect map of $P$ ). $G$ is constructed by removing from the complete graph over $U$ every edge $(\alpha, \beta)$, such that $\alpha \in X, \beta \in Y$ for some statement $\sigma=I(X, Z, Y) \in \Sigma$, and only these edges.

The closure algorithm is now clear: given $\Sigma$, it constructs $G$ by the procedure of Theorem 8 and uses graph separation to identify the elements of $\mathrm{cl}(\Sigma)$. The construction of $G$ requires $O\left(k^{2}\right)$ steps, where $k$ is the size of $\Sigma$ and $n$ is the number of variables, while to verify if a specific statement belongs to $\operatorname{cl}(\Sigma)$ requires $O(n)$ steps. The simplicity of this algorithm stems from the fact that axioms (1.a) through (1.c) and (3) are complete for fixed context statements both when interpreted as vertex separation (Theorem 8 , part 1) as well as when interpreted as conditional independence in $P D^{+}$(Theorem 8, part 2).

Theorem 7 is important by itself because it justifies the use of undirected graphs as a representation scheme for probabilistic dependencies. It allows one to choose any UG for representing dependencies and be guaranteed that the model is supported by probability theory (similar results for Directed Acyclic Graphs are presented in [13]). The converse, however, does not hold; there are many distributions that do not have a perfect representation either in DAGs or in UGs and the challenge remains to devise graphical representations that minimize this deficiency.

The construction presented in the proof of Theorem 7 [14] leads to a rather complex distribution, where the domain of each variable is unrestricted. It still 
does not guarantee that a set of dependencies and independencies represented by UGs is realizable in a more limited class of distributions such as normal or those defined on binary variables. We conjecture that these two classes of distributions are sufficiently rich to permit the consistency of undirected graph representations.

\section{Application to Bayesian networks}

A Bayesian network encodes properties of a probability distribution using a Directed Acyclic Graph (DAG). Each node $i$ in a Bayesian network corresponds to a variable $X_{i}$, a set of nodes $I$ correspond to a set of variables $X_{I}$ and $x_{i}$ is a value from the domain of $X_{i}$. Each node in the network is regarded as a storage cell for the distribution $P\left(x_{i} \mid x_{\pi(i)}\right)$ where $X_{\pi(i)}$ is a set of variables that correspond to the parent nodes $\pi(i)$ of $i$. The distribution represented by a Bayesian network is composed via

$P\left(x_{1}, \ldots, x_{n}\right)=\prod_{i=1}^{n} P\left(x_{i} \mid x_{\pi(i)}\right)$

(when $i$ has no parents, then $X_{\pi(i)}=\varnothing$ ). The role in a Bayesian network is to record a state of knowledge $P$, to provide means for updating the knowledge as new information is accumulated and to facilitate query answering mechanisms for knowledge retrieval $[22,27]$. A standard query for a Bayesian network is to find the current belief distribution of a hypothesis variable $X_{l}$, given a composed evidence set $X_{J}=x_{J}$ i.e., to compute $P\left(x_{l} \mid x_{J}\right)$ for each value of $X_{l}$ and for a given combination of values of $X_{J}$. We examine the following related problem: Given a variable $X_{k}$, a Bayesian network $D$ and the task of computing $P\left(x_{l} \mid x_{J}\right)$, determine, without resorting to numeric calculations, whether the answer to the query is sensitive to the value of $X_{k}$.

This question is an instance of the implication problem because it amounts to verifying whether the statement $I\left(X_{l}, X_{j}, X_{k}\right)$ logically follows from the set of independencies that define the topology of the DAG. Let the nodes (and variables) be arranged in a total ordering $d$ that agrees with the directionality of the DAG, namely, $i$ must precede $j$ whenever there is a link from $i$ to $j$. Let $U(i)$ stand for the set of nodes that precede $i$ in the ordering $d$. The rule of decomposition (4) implicitly encodes a set of equalities $P\left(x_{i} \mid x_{\pi(i)}\right)=P\left(x_{\imath} \mid x_{U_{(i)}}\right)$, $i=1 \ldots n$, or in our notations, a set of $n$ independence statements $I\left(X_{i}, X_{\pi(t)}, X_{U(t)}-X_{\pi(t)}\right)$. This set of statements, denoted $L$, is said to define a Bayesian network and is called a recursive basis. Thus, the problem at hand is to determine whether $I\left(X_{l}, X_{J}, X_{k}\right)$ logically follows from $L$. If it does, then $P\left(x_{l} \mid x_{J}\right)=P\left(x_{l} \mid x_{J}, x_{k}\right)$ for all instantiations $x_{l}, x_{k}$ and $x_{J}$, hence the value of $X_{k}$ will not affect the computation. If the statement does not follow, then the value of $X_{k}$ may have an effect on $P\left(x_{l} \mid x_{J}\right)$. 
THEOREM $9[13,40]$

An independence statement $\sigma$ logically follows from a recursive basis $L$ iff $\sigma$ can be derived from $L$ using axioms (1).

Theorem 9 provides a characterization of all statements that logically follow from $L$, whereas [29] also establishes a compact representation of these statements, based on a graphical criterion called $d$-separation. [15] employs $d$-separation to facilitate a linear time (in the number of links) implication algorithm for inputs forming a recursive basis. This result is analogous to Theorem 8 , since it provides a compact representation of the closure of $L$ (via a DAG) and a linear time algorithm to determine whether a statement belongs to the closure.

Theorem 9 assumes that $L$ contains only predecessor independencies of the form $I\left(X_{i}, X_{\pi(i)}, X_{U(i)}-X_{\pi(i)}\right)$. Occasionally, however, we are in possession of stronger forms of independence relationships, in which case additional independencies should be read off the DAG. A common example is the case of a variable that is functionally dependent on its corresponding parents in the DAG (deterministic variable [33]). The existence of such a variable $X_{i}$ could be encoded in $L$ by a statement of global independence $I\left(X_{t}, X_{\pi(t)}, X_{U}-X_{\pi(l)}-X_{i}\right)$ asserting that conditioned on $x_{\pi(l)}, X_{i}$ is independent of all other variables, not merely of its predecessors $\left(X_{U(t)}\right)$. A set combining predecessor independencies and global independencies is called a functional basis.

\section{THEOREM $10[11,15]$}

An independence statement $\sigma$ logically follows from a functional basis $L$ iff $\sigma$ can be derived from $L$ using axioms (1).

[15] shows that the independencies implied by a functional basis can be identified in linear time using an enhanced version of $d$-separation, named D-separation.

\section{Discussion}

This paper establishes a partial axiomatic characterization of conditional independence. This characterization, aside from its use in inference systems, also highlights plausible lines of reasoning that would otherwise be hidden in numerical calculations. Our axioms could serve as building blocks of systems that provide qualitative explanations as to why certain facts were or were not taken into account in a given computation. For example, the weak union axiom can be phrased verbally to read, "if two items together are irrelevant then learning one of them leaves the other still irrelevant". A numeric representation of this inference, however, involves several equations that hide the intuition that so strongly supports this inference. Finding an axiomatic basis for probabilistic 
independence, which is the main contribution of this paper, also plays a role in determining an appropriate uncertainty calculus. The axiom system clearly establishes (1) the qualitative relationships that are guaranteed to be preserved by adhering to the rules of probability calculus, and (2) the qualitative relationships that are in danger of being compromised as a result of choosing an alternative calculus.

\section{Acknowledgements}

We thank Ron Fagin for pointing out the usefulness of the notion of Armstrong relation and Norman Dalkey, Azaria Paz and Thomas Verma for many useful discussions on the properties of dependency models.

\section{References}

[1] S. Andreassen, M. Woldbye, B. Falck and S.K. Andersen, MUNIN - A causal probabilistic network for interpretation of electromyographic findings, Proc. 10th Int. Joint Conf. on AI (IJCAI-87), Milan, Italy (1987) pp. 366-372.

[2] C. Beeri, On the membership problem for functional and multivalued dependencies in relational databases, ACM Trans. Database Systems 5, no. 3 (Sept. 1980) 241-249.

[3] C. Beeri, R. Fagin and J.H. Howard, A complete axiomatization of functional dependencies and multivalued dependencies in database relations, Proc. ACM SIGMOD Int. Conf. on Management of Data, Toronto, Canada (1977) pp. 47-61.

[4] M. Ben-Bassat, R.W. Carlson, V.K. Puri, E. Lipnic, L.D. Portigal and M.H. Weil, Pattern-based interactive diagnosis of multiple disorders: The MEDAS system, IEEE Trans. Pattern Anal. Machine Intelligence PAMI-2 (1980) 148-160.

[5] H.M. Cramér, Mathematical methods of statistics (Princeton University Press, Princeton, N.J., 1946).

[6] A.P. Dawid, Conditional independence in statistical theory (with discussion), J. Roy. Statist. Soc. B41 (1979) 1-31.

[7] R.O. Duda, P.E. Hart, P. Barnett, J. Gaschnig, K. Konolige, R. Reboh and J. Slocum, Development of the PROSPECTOR consultant system for mineral exploration, Final Report for SRI Projects 5821 and 6915, Artificial Intelligence Center, SRI International (1978).

[8] R. Fagin, Functional dependencies in a relational database and propositional logic, IBM J. Res. Dev. 21 (1977) 534-544.

[9] R. Fagin, Multivalued dependencies and a new form for relational databases, ACM Trans. on Database Systems 2 (1977) 262-278.

[10] R. Fagin, Horn clauses and database dependencies, JACM 29 (1982) 952-985.

[11] D. Geiger, Thesis, UCLA, Cognitive Systems Laboratory, Technical Report R-102 (Dec. 1987).

[12] D. Geiger, A. Paz and J. Pearl, Axioms and algorithms for inferences involving probabilistic independence, UCLA, Cognitive Systems Laboratory, Technical Report R-119 (1988) to appear in: Information and Computation.

[13] D. Geiger and J. Pearl, On the logic of causal models, in: Proc. 4th Workshop on Uncertainty in AI, Minneapolis, Minnesota (Aug. 1988) pp. 136-147. 
[14] D. Geiger and J. Pearl, Logical and algorithmic properties of conditional independence and qualitative independence, UCLA, Cognitive Systems Laboratory, Technical Report R-97 (March 1988).

[15] D. Geiger, T. Verma and J. Pearl, Identifying independence in Bayesian networks, UCLA, Cognitive Systems Laboratory, Technical Report R-116 (1989), to appear in Networks.

[16] R. Howard and J.E. Matheson, Influence diagrams, in: Principles and Applications of Decision Analysis, Menlo Park, CA, Strategic Decision Group (1981).

[17] D. Hunter, Graphoids, semi-graphoids, and ordinal conditional independence, in preparation (1988).

[18] V. Isham, An introduction to spatial point processes and Markov random fields, Int. Statist. Rev. 49 (1981) 21-43.

[19] D.A. Kenny, Correlation and causality (Wiley, New York, 1979).

[20] H. Kiiveri, T.P. Speed and J.B. Carlin, Recursive causal models, J. Austral. Math. Soc. 36 (1984) 30-52.

[21] S.L. Lauritzen, Lectures on contingency tables, 2nd ed. (University of Aalborg Press, Aalborg, Denmark, 1982).

[22] S.L. Lauritzen and D. Spiegelhalter, Local computation with probabilities on graphical structures, and their application to expert systems (with discussion), J. Roy. Statist. Soc. B50 (1988) 157-244.

[23] D.S. Parker and K. Parsaye-Ghomi, Inferences involving embedded multivalued dependencies and transitive dependencies, in: Proc. ACM SIGMOD Int. Conf. on Management of Data (1980) pp. 52-57.

[24] A. Paz, Membership algorithm for marginal independence, UCLA, Cognitive Systems Laboratory, Technical Report R-117 (September 1988).

[25] A. Paz, A full characterization of pseudographoids in terms of families of undirected graphs, UCLA, Cognitive Systems Laboratory, Technical report R-95 (September 1987).

[26] J. Pearl, Markov and Bayes networks: A comparison of two graphical representations of probabilistic knowledge, UCLA, Cognitive Systems Laboratory, Technical Report R-46 (October 1986). Also in [28] (chapter 3).

[27] J. Pearl, Fusion, propagation and structuring in belief networks, Artificial Intelligence 29 (1986) 241-288.

[28] J. Pearl, Probabilistic reasoning in intelligent systems: Networks of plausible inference (Morgan Kaufmann, San Mateo, 1988).

[29] J. Pearl, D. Geiger and T. Verma, The logic of influence diagrams, to appear in: Influence Diagrams, Belief Nets and Decision Analysis, eds. R.M. Oliver and J.Q. Smith (Wiley, 1989). A shorter version in Kybernetika 25, no. 2 (1989) 33-44.

[30] J. Pearl and A. Paz, Graphoids: A graph-based logic for reasoning about relevance relations, Advances in Artificial Intelligence - II, eds. B. Du Boulay et al. (North-Holland, Amsterdam, 1987) pp. 357-363.

[31] J. Pearl and T. Verma, The logic of representing dependencies by directed acyclic graphs, Proc. $A A A-I$, Seattle, Washington (July 1987) pp. 374-379.

[32] Y. Sagiv and S. Walecka, Subset dependencies and completeness results for a subset of EMVD, JACM 29 (1982) pp. 103-117.

[33] R. Shachter, Probabilistic inference and influence diagrams, Oper. Res. 36 (1988) 589-604.

[34] P. Shenoy and G. Shafer, Propagating belief functions in qualitative Markov trees, Int. J. Approximate Reasoning 1 (1989) 349-400.

[35] J.O. Smith, Influence diagrams for statistical modeling, Technical Report 117, Department of Statistics, University of Warwick, Coventry, UK.

[36] W. Spohn, Stochastic independence, causal independence, and shieldability, J. Philos. Logic 9 (1980) 371-397. 
[37] W. Spohn, Ordinal conditional functions: A dynamic theory of epistemic states, Causation in Decision, Belief Change, and Statistics, vol. 2, eds. W.L. Harper and B. Skyrms (1988) pp. 105-134.

[38] M. Studeny, Attempts at axiomatic description of conditional independence, Workshop on Uncertainty in Expert Systems, Alsovice, Chechoslovakia (1988).

[39] M. Vardi, Fundamentals of dependency theory, in: Trends in Theoretical Computer Science, ed. E. Borger (Computer Science Press, Rockville, MD, 1988) pp. 171-224.

[40] T. Verma and J. Pearl, Causal networks: Semantics and expressiveness, in: Proc. 4th Workshop on Uncertainty in AI, Minneapolis, Minnesota (1988) pp. 352-358.

[41] N. Wermouth and S.L. Lauritzen, Graphical and recursive models for contingency tables, Biometrika 70 (1983) 537-552. 